\title{
Simulador didático para análise dos transientes de ondas de tensão em uma linha de transmissão similar à transmissão e reflexão de ondas eletromagnéticas
}

Didactic simulator for analysis of transients of voltage waves in a transmission line similar to the principle of reflection and transmission of electromagnetic waves

Francisco Marks $\mathrm{Cruz}^{1}$, Adriano Aron Freitas de Moura ${ }^{* 20}$, Ailson Pereira de Moura ${ }^{3}$, Ednardo Pereira da Rocha ${ }^{2}$, Daniel Carlos de Carvalho Crisóstomo ${ }^{4}$, Herick Talles Queiroz Lemos ${ }^{2}$

\author{
${ }^{1}$ Instituto Federal de Educação, Ciência e Tecnologia do Ceará, Maranguape, CE, Brasil \\ ${ }^{2}$ Universidade Federal Rural do Semi-Árido, Mossoró, RN, Brasil \\ ${ }^{3}$ Universidade Federal do Ceará, Fortaleza, CE, Brasil \\ ${ }^{4}$ Universidade Potiguar, Mossoró, RN, Brasil
}

Recebido em 25 de Maio, 2019. Aceito em 15 de Junho, 2019.

\begin{abstract}
O modelo de educação nas últimas décadas passou por mudanças consideráveis do ponto de vista didático. Com o avanço da tecnologia, a velocidade de obtenção de informações aumenta e o uso de ferramentas que permitem a visualização dinâmica de conteúdos torna-se um forte aliado ao processo de ensino-aprendizagem. Este trabalho trata da criação de uma ferramenta interativa para visualizar a propagação de ondas de tensão em uma linha de transmissão similar ao que acontece à transmissão e reflexão de ondas eletromagnéticas que incidem em meios dielétricos diferentes, permitindo a inserção de dados pelo usuário, a fim de facilitar a consolidação do conhecimento. O modelo foi criado a partir do GUIDE do MATLAB, que possui uma interface gráfica com botões de ação que ao serem clicados executam ações como calcular e exibir o gráfico animado. É importante ressaltar que a criação do programa, além de facilitar o entendimento, também permite maior familiarização com o software de simulação computacional e com os parâmetros utilizados na análise transitória de linhas de transmissão. Assim, a análise dos transitórios de ondas de tensão em linhas de transmissão, que consiste em uma difícil área de aprendizagem, devido ao seu caráter teórico, torna-se de fácil assimilação com o uso dessa ferramenta didático-computacional.
\end{abstract}

Palavras-chave: Ondas viajantes, MATLAB GUIDE, simulação computacional, animação no MATLAB.

The model of education in the last decades has undergone considerable changes from a didactic point of view. With the advancement of technology, the speed of getting information increases and the use of tools that allow the dynamic visualization of contents becomes a strong ally to the teaching-learning process. This work deals with the creation of an interactive tool to visualize the propagation of voltage waves in a transmission line similar to what happens to the transmission and reflection of electromagnetic waves that affect different dielectric means, allowing the insertion of data by the user, to facilitate the consolidation of knowledge. We created the model from the MATLAB GUIDE, which has a graphical interface with action buttons that, when clicked, perform actions such as calculating and displaying the animated graphic. It is important to emphasize that the creation of the program, besides facilitating the understanding, also allows greater familiarization with the computer simulation software and with the parameters used in the transient analysis of transmission lines. Thus, the analysis of transients of voltage waves in transmission lines, which comprises a difficult learning area, for its theoretical nature, becomes easy to assimilate with the use of this didactic-computational tool.

Keywords: Traveling waves, MATLAB GUIDE, computer simulation, animation in MATLAB.

\section{Introdução}

A cada dia percebe-se o aumento na velocidade de aquisição das informações. A internet proporciona a conexão quase instantânea de acontecimentos em todo o globo. Dentro desta perspectiva, os computadores e dispositivos portáteis são responsáveis por grande parcela da disse-

*Endereço de correspondência: adrianoaron@ufersa.edu.br minação de informações. Desta forma, a interatividade com softwares pode ser uma forte aliada ao processo de ensino-aprendizado.

Dentro desta perspectiva, a criação de uma interface gráfica com a possibilidade de inserção de dados diretamente pelo usuário e exibição de animações mostrando o comportamento das ondas viajantes em um sistema elétrico, mostra-se uma alternativa didática e bastante 
auxiliar, tanto na apresentação de um novo conteúdo quanto na consolidação do conhecimento segundo [1].

O comportamento das faltas ou chaveamentos iniciados no transitório em sistemas de potência é extremamente importante, não apenas do ponto de vista da proteção, controle e qualidade da energia, como sobretudo do ponto de vista de propostas educacionais.

Os conceitos de transitórios de tensão são similares ao comportamento de ondas eletromagnéticas, quando incidem em meios dielétricos diferentes e são de difícil assimilação e visualização, podendo ser melhor compreendidos usando técnicas de animação gráfica, que ajudam a explicar o complexo comportamento espaço-tempo desses transitórios durante faltas ou outras perturbações.

Tais perturbações têm como consequência a geração de perdas econômicas muito importantes, tanto para os usuários quanto para as empresas que comercializam a energia, conforme [2].

Embora os sistemas de potência operem em regime permanente na maior parte do tempo, é necessário que eles suportem a pior situação a qual possam se submeter, normalmente encontrada durante situações de transitório [3]. Há duas maneiras de estudo dos transitórios em sistemas elétricos de potência:

- Simulação analógica: o sistema é representado por Analisadores Transitórios de Redes (TNA's - Transient Network Analyzer), o qual é uma reprodução física em escala reduzida dos componentes do sistema de potência;

- Simulação computacional: os componentes do sistema de potência são representados através de modelos matemáticos implementados computacionalmente.

Devido ao nível de complexidade do equacionamento das linhas de transmissão, pelo fato de envolver sistemas de equações diferenciais, a possibilidade da criação de uma ferramenta didática no âmbito da engenharia apresenta-se como uma importante ferramenta.

Os programas de simulação no domínio do tempo, no geral, calculam somente a tensão nos terminais da linha, enquanto a animação do transitório possibilita sua visualização ao longo da linha.

Na próxima seção serão vistos os modelos de linhas de transmissão, os quais foram utilizados na modelagem matemática do problema, assim como na escolha das configurações que compõem o simulador.

\section{Modelos de linhas de transmissão}

Ao ser percorrida por corrente alternada, uma linha de transmissão (LT) produz uma queda de tensão em seus terminais devido à impedância oferecida pela linha. Para modelagem dessa característica, existem três modelos mais difundidos que oferecem aproximações para três situações construtivas, baseadas no comprimento e nível de tensão da LT.

\subsection{Linha Curta}

Uma linha de transmissão é considerada curta quando possui comprimento inferior a $80 \mathrm{~km}$, ou se seu nível de tensão máximo é $69 \mathrm{kV}$ como observa [3]. Neste modelo, a capacitância pode ser desprezada sem a produção de erros consideráveis. A impedância da linha pode ser obtida multiplicando-se a impedância série por unidade de comprimento pelo comprimento da linha, conforme (1).

$$
Z=(r+j \omega L) l
$$

Em (1), $r$ e $L$ são a resistência e a indutância por fase por unidade de comprimento, respectivamente, e $l$ é o comprimento da linha.

O modelo de linha curta é mostrado na Figura 1. O subscrito "S"representa os valores enviados (sending), ou seja, os valores disponibilizados à linha, seja de corrente, tensão ou potência. Já o sobrescrito "R"é utilizado devido ao termo em inglês (receiving), ou seja, recebido, que é o valor verificado na carga.

De acordo com as Leis de Kircchoff, após a análise da malha, pode-se chegar à formulação básica das linhas curtas, conforme (2), (3) e 4):

$$
\begin{gathered}
V_{s}-Z \cdot I_{R}-V_{R}=0 \\
V_{s}=Z \cdot I_{R}+V_{R}
\end{gathered}
$$

Onde $Z$ é a impedância série da linha e:

$$
I_{s}=I_{R}
$$

A corrente $I_{R}$ foi convenientemente colocada do lado direito de (3) para explicitar os parâmetros no receptor como resultado do disponibilizado à linha.

As equações (3) e (4) podem ser representadas através do modelo do quadripolo ABCD, conforme (5).

$$
\left[\begin{array}{l}
V_{s} \\
I_{s}
\end{array}\right]=\left[\begin{array}{ll}
1 & Z \\
0 & 1
\end{array}\right]\left[\begin{array}{l}
V_{R} \\
I_{R}
\end{array}\right]
$$

\subsection{Linha Média}

Conforme a linha aumenta, as mudanças na corrente se tornam consideráveis e a capacitância shunt deve ser considerada. Uma LT é considerada média quando seu comprimento está entre 80 e $250 \mathrm{~km}$ à $60 \mathrm{~Hz}$, conforme $[4]$.

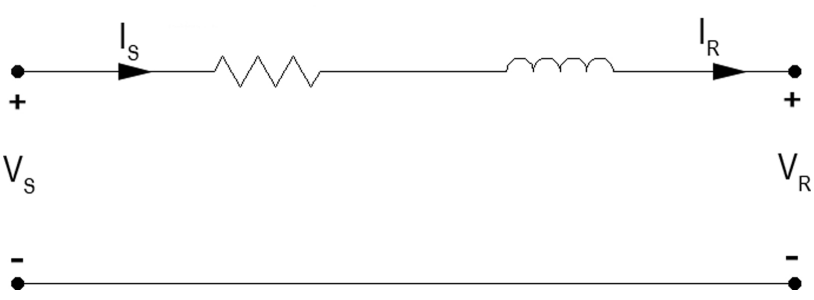

Figura 1: Modelo de linha curta. 
Neste caso, é comum utilizar uma impedância capacitiva dividida nas extremidades da linha. Essas impedâncias são normalmente expressas em termos de admitâncias. O circuito equivalente, chamado "circuito $\pi$ nominal", é mostrado na Figura 2.

A partir das Leis de Kirchhoff, obtém-se para a seguinte relação para a tensão no terminal de envio:

$$
V_{S}=\left(\frac{Z Y}{2}+1\right) V_{R}+Z I_{R}
$$

Seguindo o mesmo princípio, a corrente nesse terminal é definida de acordo com (7).

$$
I_{S}=\left(1+\frac{Z Y}{4}\right) Y V_{R}+\left(1+\frac{Z Y}{2}\right) I_{R}
$$

As equações (6) e (7) podem ser reescritas na forma de um quadripolo ABCD, conforme (8).

$$
\left[\begin{array}{c}
V_{S} \\
I_{S}
\end{array}\right]=\left[\begin{array}{cc}
1+\frac{Z Y}{2} & Z \\
\left(1+\frac{Z Y}{4}\right) Y & 1+\frac{Z Y}{2}
\end{array}\right]\left[\begin{array}{c}
V_{R} \\
I_{R}
\end{array}\right]
$$

\subsection{Linha Longa}

O modelo de linha longa (Figura 3), ou de parâmetros distribuídos, considera que as características capacitivas e indutivas da linha não estão concentradas nas suas extremidades, como nas linhas médias, mas distribuídos ao longo dela, observa [5].

Neste modelo, $\mathrm{R}$ e L representam, respectivamente, a resistência e a indutância por unidade de comprimento de cada segmento de linha representado por um único circuito, chamado circuito $\pi$. Os parâmetros G e C são, respectivamente, a condutância e a capacitância do segmento de linha.

Cada segmento que compõe a linha tem comprimento infinitesimal $\Delta x$ e produz entre suas extremidades as diferenças de tensão e de corrente $\Delta V$ e $\Delta I$ respectivamente.

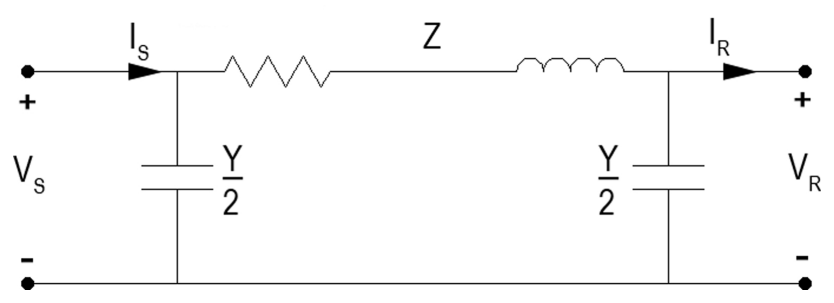

Figura 2: Modelo de linha média.
Além disso, a impedância série e admitância shunt de cada seguimento são $z \Delta x$ e $y \Delta x$ respectivamente.

Para um segmento localizado a uma distância $x$ do terminal de receptor, a queda de tensão $\Delta V$ é produzida quando uma corrente $I$ percorre a impedância série desse segmento, ou seja, $z \Delta x$. Desse fato, decorre que a extremidade do segmento no lado do terminal de envio possui tensão $V+\Delta V$, a extremidade no lado do terminal receptor possui tensão $V$ e a queda de tensão no segmento é $z \Delta x I$, conforme (9).

$$
\Delta V=I z \Delta x
$$

Da equação (9), verifica-se que a taxa de variação da tensão em função da distância $x$ é dada por:

$$
\frac{\Delta V}{\Delta x}=z I
$$

$\mathrm{E}$, quando $\Delta x \rightarrow 0$, o limite do quociente de 10 se torna:

$$
\frac{d V}{d x}=z I
$$

Uma relação análoga pode ser desenvolvida para a corrente que flui em cada segmento de linha. Para um segmento localizado a uma distância $x$ do terminal receptor, a diferença de corrente $\Delta I$ entre as extremidades desse segmento corresponde à parcela da corrente de entrada que flui por sua admitância shunt. Sendo assim, na extremidade do lado do terminal de envio desse segmento flui uma corrente $I+\Delta I$ e na extremidade do lado do terminal receptor flui uma corrente $I$.Portanto:

$$
\Delta I=y \Delta x V
$$

E, adotando os mesmos passos para obter (11), deduzse para a corrente:

$$
\frac{d I}{d x}=V y
$$

Derivando 11 e 13 em relação a $x$, obtém-se o seguinte:

$$
\begin{aligned}
& \frac{d^{2} V}{d x^{2}}=z \frac{d I}{d x} \\
& \frac{d^{2} I}{d x^{2}}=y \frac{d V}{d x}
\end{aligned}
$$

Substituindo 11 e 13 em (14) e 15 respectivamente, obtêm-se (16) e (17).

$$
\frac{d^{2} V}{d x^{2}}=y z V
$$

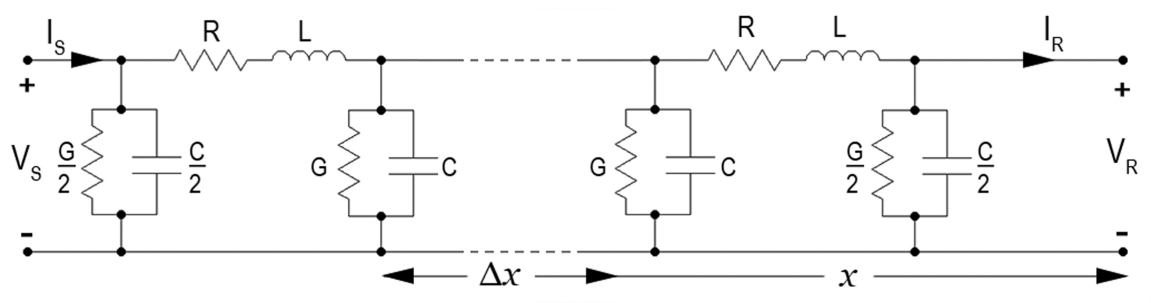

Figura 3: Modelo de linha longa. 


$$
\frac{\mathrm{d}^{2} I}{d x^{2}}=y z I
$$

Como $V$ e $I$ são funções de $x$, 16 e 17) são duas equações diferenciais de segunda ordem, cuja solução geral é dada por:

$$
V(x)=A_{1} e^{\gamma x}+A_{2} e^{-\gamma x}
$$

Onde $\gamma$, conhecido como constante de propagação, é uma expressão complexa dada por:

$$
\gamma=\alpha+j \beta=\sqrt{z y}=\sqrt{(R+j \omega L)(G+j \omega C)}
$$

A parte real, $\alpha$, é conhecida como constante de atenuação, e a componente imaginária, $\beta$, é conhecida como constante de fase, sendo dada em radiano por unidade de comprimento.

A solução de 13 é dada em 20 .

$$
I(x)=\frac{\gamma}{z} \frac{d V(x)}{d x}=\frac{\gamma}{z}\left(A_{1} e^{\gamma x}-A_{2} e^{-\gamma x}\right)
$$

A equação 20 pode ainda ser expressa por:

$$
I(x)=\sqrt{\frac{y}{z}}\left(A_{1} e^{\gamma x}-A_{2} e^{-\gamma x}\right)
$$

Sabendo-se que a impedância característica, $Z_{c}$, é dada por:

$$
Z_{C}=\sqrt{\frac{Z}{y}}
$$

A corrente ao longo da linha pode ser expressa em função da impedância característica conforme a equação (23).

$$
I(x)=\frac{1}{Z_{C}}\left(A_{1} e^{\gamma x}-A_{2} e^{-\gamma x}\right)
$$

As constantes $A_{1}$ e $A_{2}$ podem ser obtidas com base nas condições iniciais do sistema. Para $x=0, V=V_{R}$ e $I=I_{R}$. Substituindo-se estes valores em (18) e 23), obtém-se:

$$
\begin{gathered}
V_{R}=A_{1}+A_{2} \\
I_{R}=\frac{I}{\sqrt{z / y}}\left(A_{1}-A_{2}\right)
\end{gathered}
$$

A solução das equações (24) e (25) em termos de $A_{1}$ e $A_{2}$, seguida da transformação das equações (18) e 23) para a forma hiperbólica, resulta nas equações que representam a tensão e corrente ao longo da linha, isto é:

$$
\begin{gathered}
V(x)=V_{R} \cosh \gamma x+I_{R} Z_{C} \operatorname{senh} \gamma x \\
I(x)=I_{R} \cosh \gamma x+\frac{V_{R}}{Z_{C}} \operatorname{senh} \gamma x
\end{gathered}
$$

Para $x=l$, obtém-se a tensão e corrente na barra de envio:

$$
\begin{gathered}
V_{S}=V_{R} \cosh \gamma l+I_{R} Z_{C} \operatorname{senh} \gamma l \\
I_{S}=\frac{V_{R}}{Z_{C}} \operatorname{senh} \gamma l+I_{R} \cosh \gamma l
\end{gathered}
$$

Assim, na forma matricial, a tensão e a corrente podem ser representadas conforme 300 .

$$
\left[\begin{array}{l}
V_{s} \\
I_{s}
\end{array}\right]=\left[\begin{array}{cc}
\cosh \gamma l & Z_{C} \operatorname{senh} \gamma l \\
\frac{1}{Z_{C}} \operatorname{senh} \gamma l & \cosh \gamma l
\end{array}\right]\left[\begin{array}{c}
V_{R} \\
I_{R}
\end{array}\right]
$$

O modelo de linha longa também é aplicável aos modelos mencionados anteriormente (de linha curta e média) quando aplicadas as devidas considerações matemáticas, visto que se trata do modelo mais completo para representação de linhas de transmissão.

\section{Processo de desenvolvimento do simulador}

O desenvolvimento do simulador foi realizado em duas etapas. A primeira, mais básica, consistiu na concepção da interface gráfica tenho em vista a abordagem de ondas viajantes com enfoque educacional. A segunda, de maior complexidade, consistiu na formulação matemática das ondas viajantes em quatro topologias (configurações) de sistemas elétricos e posterior implementação computacional. Apesar de o tratamento matemático mais preciso das ondas viajantes ser definido pelas equações de Maxwell [6], neste trabalho utilizou-se uma abordagem simplificada para a modelagem do fenômeno, conforme [7], visto que o simulador proposto tem cunho educacional. Para representação das linhas de transmissão, foi utilizado o modelo com parâmetros distribuídos (linha longa) discutido na Seção 2.

\subsection{Recursos Utilizados}

A ferramenta GUI do MATLAB, chamada GUIDE, foi utilizada para a programação e criação gráfica, auxiliando na construção das interfaces gráficas para interação com o utilizador. Foi utilizado ainda softwares de edição de imagens para criação de imagens para cada uma das configurações de sistemas de potência utilizadas no simulador.

\subsection{Definição dos Modelos}

Os modelos foram criados em ordem de complexidade crescente, observando-se a possibilidade de aprendizado pelo corpo discente, por isso, limitou-se o número de barras em até quatro e de geração em apenas uma, para que seja viável e compreensível, do ponto de vista de um estudante de graduação, o comportamento das ondas viajantes em uma linha de transmissão.

O simulador é composto por quatro configurações com caixas para entrada dos dados, botões de seleção e de execução. Os dados de entrada serão inseridos via teclado numérico e suas ações feitas através de cliques nos botões específicos de ação. 


\subsection{Configuração 1}

A Configuração 1, conforme Figura 4, possui o modelo mais simples para estudo do perfil de ondas viajantes numa linha de transmissão. Essa configuração é composta por de uma única geração alimentando a barra 1. As ondas viajantes percorrerão o caminho 1-2, realizando as transmissões e reflexões sucessivas.

A dedução matemática da Configuração 1 foi feita conforme os coeficientes especificados na Figura 5.

As equações (31) e (32) descrevem as ondas de tensão nas barras 1 e 2 em função dos coeficientes apresentados na Figura 5.

$$
\begin{gathered}
v_{1}(t)=v(t)+\rho_{t 1} \sum_{\alpha=0}^{\infty} \rho_{r 1}^{\alpha} \rho_{r 2}^{\alpha+1} v(t-2(\alpha+1) \tau) \\
v_{2}(t)=\rho_{t 2} \sum_{\alpha=0}^{\infty}\left(\rho_{r 1} \rho_{r 2}\right)^{\alpha} v(t-(2 \alpha+1) \tau)
\end{gathered}
$$

Onde, $\rho_{r i-j}$ é o coeficiente de reflexão entre a barra $i-j$, definido conforme $(33)$ e $\rho_{t i-j}$ é o coeficiente de transmissão, conforme (34).

$$
\begin{gathered}
\rho_{r i-j}=\frac{Z_{j}-Z_{i}}{Z_{i}+Z_{j}} \\
\rho_{t i-j}=\rho_{r i-j}+1
\end{gathered}
$$

O tempo de deslocamento, $\tau_{i}$ indica o tempo que a onda viajante no trecho $i$ leva para percorrer a linha. Ele é definido como a razão entre o comprimento da linha, $l_{i}$, e a velocidade de propagação da onda no meio, que será adotada como a velocidade da luz, c portanto:

$$
\tau_{i}=\frac{l_{i}}{c}
$$

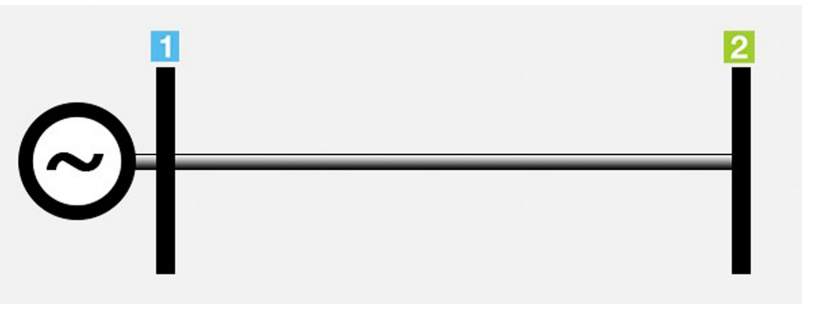

Figura 4: Configuração 1.

(1)

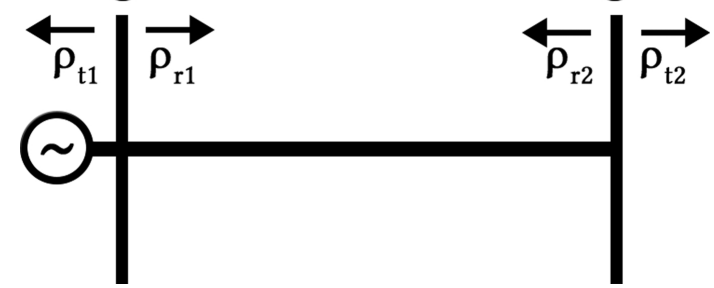

Figura 5: Coeficientes utilizados na Configuração 1.

\subsection{Configuração 2}

A configuração 2, conforme Figura 6, possui dois trechos diferentes. Por meio da interface gráfica, o usuário poderá inserir os dados de entrada para simulação e escolher em qual trecho deseja o comportamento das ondas viajantes, isto é, entre as barras 1-2 ou 2-3.

Para a dedução matemática dos sinais de tensão foi utilizado o mesmo procedimento adotado no item anterior, considerando a simbologia adotada na Figura 7.

$\mathrm{Na}$ barra 1, foram consideradas as reflexões sucessivas entre as barras 1 e 2 , sobrepostas à transmitida do meio 2 para o 1 e suas sucessivas reflexões no meio 2. A equação (37) apresenta a dedução resultante.

$$
\begin{aligned}
& v_{1}(t)=v_{0}(t)+\rho_{t g} \rho_{r 1-2} v\left(t-2 \tau_{1}\right)+ \\
& +\rho_{t g} \rho_{r g} \rho_{r 1-2}^{2} v\left(t-4 \tau_{1}\right)+\cdots \\
& \cdots+\rho_{t g} \rho_{t 1-2} \rho_{t 2-1} v\left(t-2\left(\tau_{1}+\tau_{2}\right)\right)+ \\
& +\rho_{t g}\left(\rho_{t 1-2} \rho_{r 1-2}\right)^{2} v\left(t-4\left(\tau_{1}+\tau_{2}\right)\right)+\cdots \\
& \cdots+\rho_{t g} \rho_{r g} \rho_{r 1-2} \rho_{t 1-2} \rho_{t 2-1} v\left(t-2\left(2 \tau_{1}+\tau_{2}\right)\right)+ \\
& +\rho_{t g}\left(\rho_{r g} \rho_{r 1-2}\right)^{2} \rho_{t 1-2} \rho_{t 2-1} v\left(t-4\left(2 \tau_{1}+\tau_{2}\right)\right)+\cdots
\end{aligned}
$$

Para a barra 2, a seguinte expressão foi definida:

$$
\begin{aligned}
& v_{2}(t)=\rho_{t 1-2} \rho_{r 1-2} v\left(t-\tau_{1}\right)+\ldots \\
& +\rho_{t 1-2} \rho_{r g} \rho_{r 1-2}^{2} v\left(t-3 \tau_{1}\right)+\ldots \\
& +\rho_{t 1-2} \rho_{r g}^{2} \rho_{r 1-2}^{2} v\left(t-5 \tau_{1}\right)+\ldots \\
& +\rho_{t 2-1} \rho_{t 1-2} \rho_{r 2-1} \rho_{r f}^{2} v\left(t-\left(\tau_{1}+2 \tau_{2}\right)\right)+ \\
& +\rho_{t 2-1} \rho_{t 1-2}\left(\rho_{r 2-1} \rho_{r f}\right)^{2} v\left(t-\left(\tau_{1}+4 \tau_{2}\right)\right)+ \\
& +\rho_{t 2-1} \rho_{t 1-2}\left(\rho_{r 2-1} \rho_{r f}\right)^{3} v\left(t-\left(\tau_{1}+6 \tau_{2}\right)\right)+\ldots
\end{aligned}
$$

Para a barra 3, a seguinte expressão foi definida:

$$
\begin{aligned}
& v_{3}(t)=\rho_{t 1-2} \rho_{r 1-2} v\left(t-\left(\tau_{1}+\tau_{2}\right)\right)+ \\
& +\rho_{t 1-2} \rho_{r f} \rho_{r 2} v\left(t-\left(\tau_{1}+3 \tau_{2}\right)\right)+ \\
& +\rho_{t g} \rho_{r 1-2} \rho_{t 1-2} v\left(t-\left(3 \tau_{1}+\tau_{2}\right)\right)+\cdots
\end{aligned}
$$

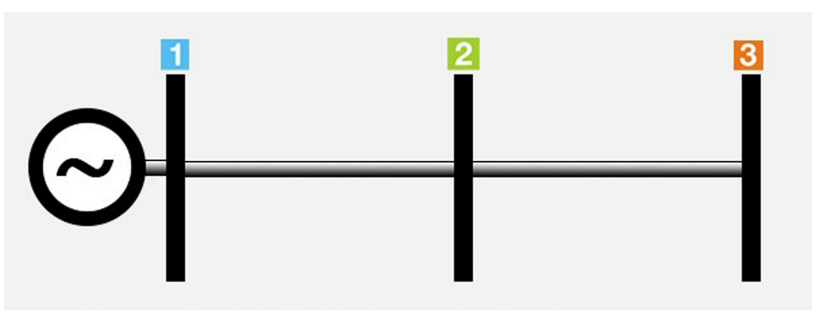

Figura 6: Configuração 2.

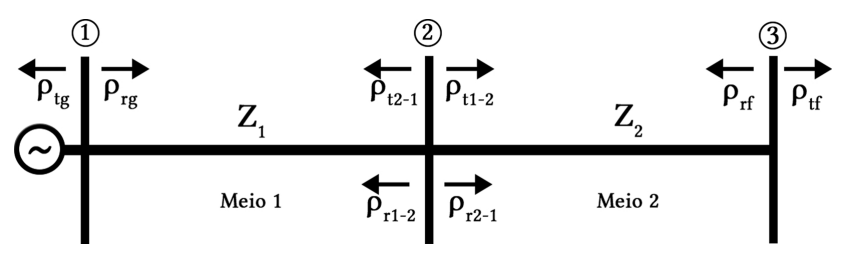

Figura 7: Coeficientes utilizados na Configuração 2. 


\subsection{Configuração 3}

A arranjo da configuração é apresentado na Figura 8. Nessa, o gerador é conectado na barra 1 e alimenta as barras 2 e 3. Nessa configuração, os efeitos da linha 1-2 são sentidos pela linha 1-3, tornando o comportamento dessa topologia mais complexo em relação aos casos anteriores.

Para a dedução matemática de cada uma das três barras desta configuração, foi utilizado o modelo matemático seguido nos casos anteriores, com a Figura 9 como referencial.

Para a barra 1, obtém-se:

$$
\begin{aligned}
& v_{1}(t)=v_{0}(t)+\rho_{t 1} \rho_{r 2-1} v\left(t-2 \tau_{1}\right)+ \\
& +\rho_{t 1} \rho_{r 1-2} \rho_{r 2-1}^{2} v\left(t-4 \tau_{1}\right)+ \\
& +\rho_{t 1} \rho_{r 1-2}^{2} \rho_{r 2-1}^{3} v\left(t-6 \tau_{1}\right)+\cdots \\
& +\rho_{t 1} \rho_{r 1-3} \rho_{r 1-3} v\left(t-2 \tau_{2}\right)+ \\
& +\rho_{t 1} \rho_{r 1-3} \rho_{r 1-3}^{2} v\left(t-4 \tau_{2}\right)+ \\
& +\rho_{t 1} \rho_{r 1-3}^{2} \rho_{r 1-3}^{3} v\left(t-6 \tau_{2}\right)+\cdots
\end{aligned}
$$

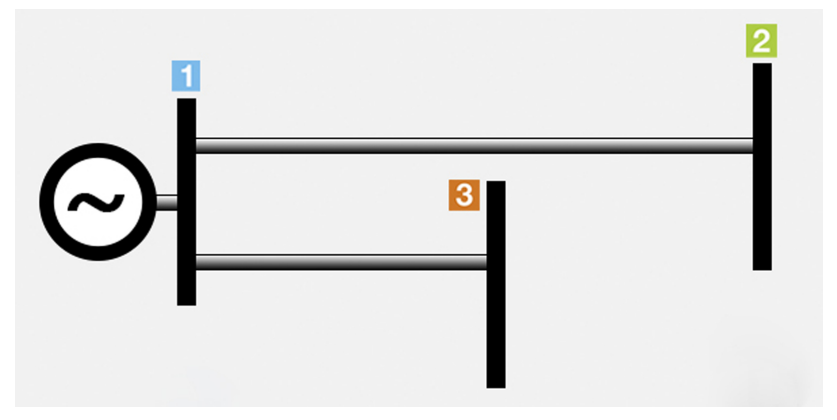

Figura 8: Configuração 3.
(1)

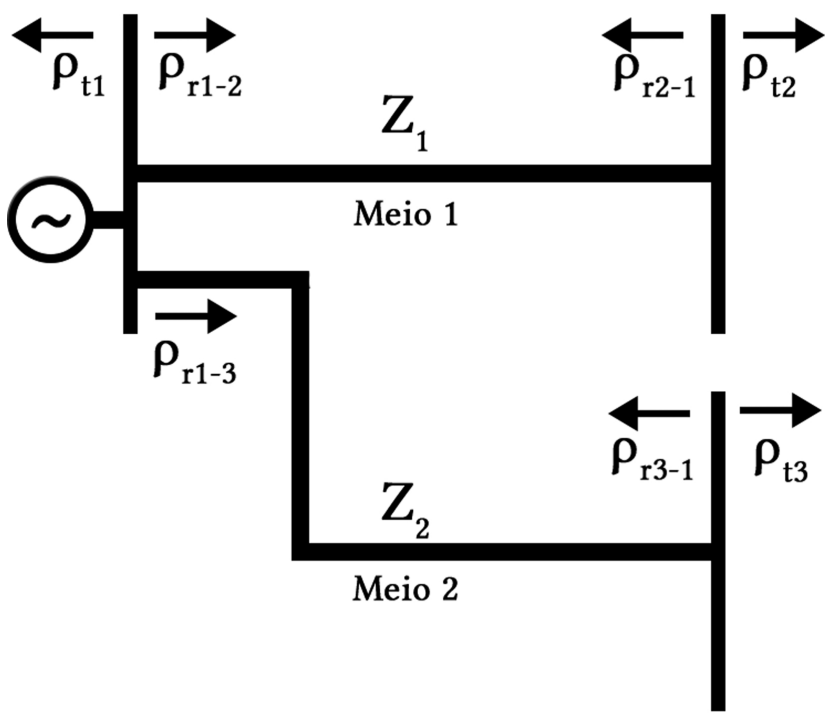

(3)
Figura 9: Coeficientes utilizados na Configuração 3.
Para a barra 2, obtém-se:

$$
\begin{aligned}
& v_{2}(t)=\rho_{t 2} v\left(t-\tau_{1}\right)+\rho_{t 2} \rho_{r 1-2} \rho_{r 2-1} v\left(t-3 \tau_{1}\right)+ \\
& +\rho_{t 2} \rho_{r 1-2}^{2} \rho_{r 2-1} v\left(t-5 \tau_{1}\right)+\cdots \\
& +\rho_{t 2} \rho_{r 1-2} \rho_{r 3-1} v\left(t-\left(\tau_{1}+\tau_{2}\right)\right)+ \\
& +\rho_{t 2} \rho_{r 1-2}^{2} \rho_{r 2-1}^{2} \rho_{r 3-1} v\left(t-2\left(\tau_{1}+\tau_{2}\right)\right)+ \\
& +\rho_{t 2} \rho_{r 1-2}^{3} \rho_{r 2-1}^{4} \rho_{r 3-1}^{2} v\left(t-3\left(\tau_{1}+\tau_{2}\right)\right)+\cdots
\end{aligned}
$$

E, para a barra 3, obtém-se:

$$
\begin{aligned}
& v_{3}(t)=\rho_{t 3} v\left(t-\tau_{2}\right)+\rho_{t 3} \rho_{r 1-3} \rho_{r 3-1} v\left(t-3 \tau_{2}\right)+ \\
& +\rho_{t 3} \rho_{r 1-3}^{2} \rho_{r 3-1} v\left(t-5 \tau_{1}\right)+\cdots \\
& +\rho_{t 2} \rho_{r 1-2} \rho_{r 3-1} v\left(t-\left(\tau_{1}+\tau_{2}\right)\right)+ \\
& +\rho_{t 2} \rho_{r 1-2}^{2} \rho_{r 2-1}^{2} \rho_{r 3-1} v\left(t-2\left(\tau_{1}+\tau_{2}\right)\right)+ \\
& +\rho_{t 2} \rho_{r 1-2}^{3} \rho_{r 2-1}^{4} \rho_{r 3-1}^{2} v\left(t-3\left(\tau_{1}+\tau_{2}\right)\right)+\cdots
\end{aligned}
$$

\subsection{Configuração 4}

A configuração 4, conforme a Figura 10, é uma composição entre as configurações 1 e 2 . A análise do seu comportamento constitui o caso mais complexo, visto que o seu arranjo é misto e possui um número mais elevado de barras. A dedução matemática seguirá o mesmo processo dos casos anteriores.

A Figura 11 apresenta os coeficientes de reflexão e transmissão envolvidos no processo de viagem das ondas na configuração 4 .

Com base na Figura 11, foram deduzidas as equações (43), 44), (44) e (45), as quais definem as ondas viajantes

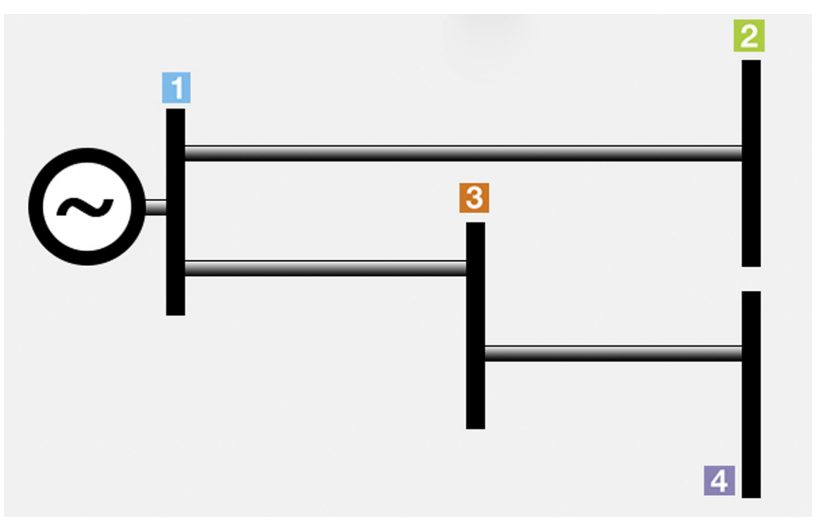

Figura 10: Configuração 4. 
(1)

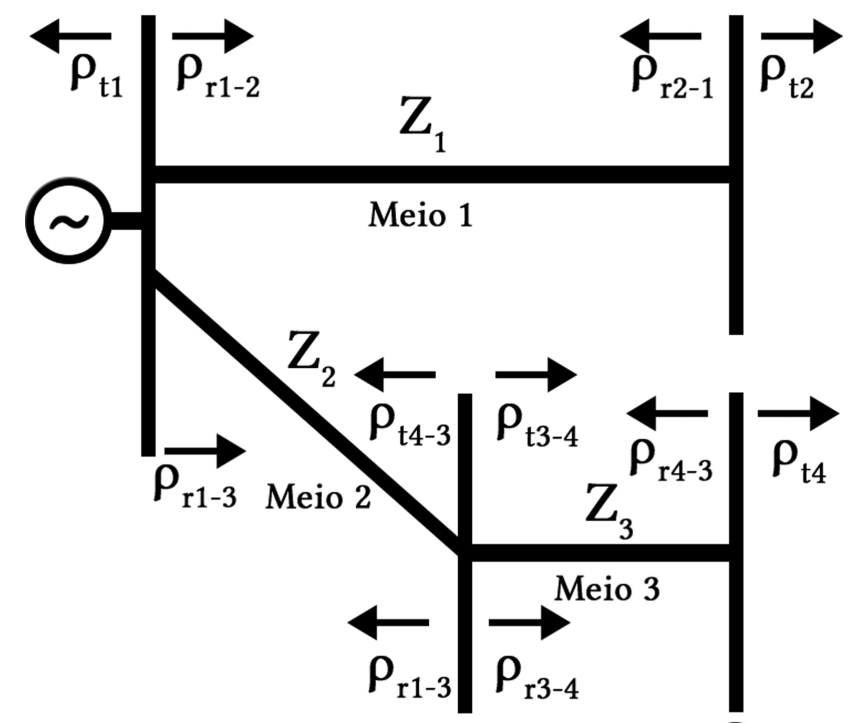

(3)

Figura 11: Coeficientes utilizados na Configuração 4.

em cada barra em função do tempo.

$$
\begin{aligned}
& v_{1}(t)=v_{0}(t)+\rho_{t 1} \rho_{r 2-1} v\left(t-2 \tau_{1}\right)+ \\
& +\rho_{t 1} \rho_{r 1-2} \rho_{r 2-1}^{2} v\left(t-4 \tau_{1}\right)+ \\
& +\rho_{t 1} \rho_{r 1-2}^{2} \rho_{r 2-1}^{3} v\left(t-6 \tau_{1}\right)+\cdots \\
& +\rho_{t 1} \rho_{r 1-3} \rho_{r 1-3} v\left(t-2 \tau_{2}\right)+ \\
& +\rho_{t 1} \rho_{r 1-3} \rho_{r 1-3}^{2} v\left(t-4 \tau_{2}\right)+ \\
& +\rho_{t 1} \rho_{r 1-3}^{2} \rho_{r 1-3}^{3} v\left(t-6 \tau_{2}\right)+\cdots \\
& +\rho_{t 1} \rho_{r 2-1}\left(\rho_{t 3-4} \rho_{r 4-3} \rho_{t 4-3}\right) \\
& \times v\left(t-2\left(\tau_{2}+\tau_{3}\right)-2 \tau_{1}\right) \\
& +\rho_{t 1} \rho_{r 2-1}\left(\rho_{t 3-4} \rho_{r 4-3} \rho_{t 4-3}\right)^{2} \\
& \left.\times v\left(t-4\left(\tau_{2}+\tau_{3}\right)-2 \tau_{1}\right)\right) \\
& +\rho_{t 1} \rho_{r 2-1}\left(\rho_{t 3-4} \rho_{r 4-3} \rho_{t 4-3}\right)^{3} \\
& \left.\times v\left(t-6\left(\tau_{2}+\tau_{3}\right)-2 \tau_{1}\right)\right)+\cdots
\end{aligned}
$$

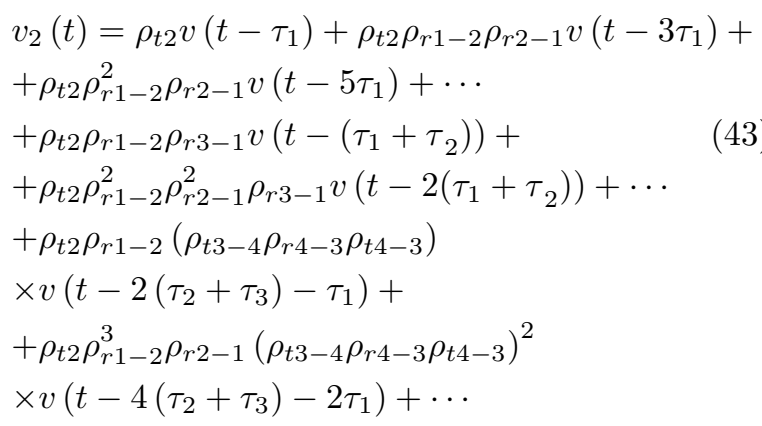

$$
\begin{aligned}
& v_{3}(t)=\rho_{t 3} v\left(t-2 \tau_{2}\right)+\rho_{t 3} \rho_{r 1-3} \rho_{r 3-1} v\left(t-3 \tau_{2}\right)+ \\
& +\rho_{t 3} \rho_{r 1-3}^{2} \rho_{r 3-1} v\left(t-5 \tau_{2}\right)+\cdots \\
& +\rho_{t 2} \rho_{r 1-2} \rho_{r 3-1} v\left(t-\left(\tau_{1}+\tau_{2}\right)\right)+ \\
& +\rho_{t 2} \rho_{r 1-2}^{2} \rho_{r 2-1}^{2} \rho_{r 3-1} v\left(t-2\left(\tau_{1}+\tau_{2}\right)\right)+\cdots \\
& +\rho_{t 3-4} \rho_{t 4-3} v\left(t-\tau_{2}-2 \tau_{3}\right)+ \\
& +\rho_{t 3-4} \rho_{t 4-3} \rho_{r 4-3}^{2} \rho_{r 3-4} v\left(t-\tau_{2}-4 \tau_{3}\right)+ \\
& +\rho_{t 3-4} \rho_{t 4-3} \rho_{r 4-3}^{3} \rho_{r 3-4}^{2} v\left(t-\tau_{2}-6 \tau_{3}\right)+\cdots
\end{aligned}
$$

$$
\begin{aligned}
& v_{4}(t)=\rho_{t 4} \rho_{r 2-1} \rho_{r 1-2} \rho_{t 3-4} v\left(t-2 \tau_{1}-\tau_{2}-\tau_{3}\right)+ \\
& +\rho_{t 4} \rho_{t 3-1} \rho_{t 1-3}\left(\rho_{r 2-1} \rho_{r 1-2} \rho_{t 3-4}\right)^{2} \\
& \times v\left(t-4 \tau_{1}-3 \tau_{2}-3 \tau_{3}\right)+\cdots \\
& +\rho_{t 4} \rho_{t 3-4} \rho_{r 3-1} v\left(t-\left(\tau_{2}+\tau_{3}\right)\right)+ \\
& +\rho_{t 4} \rho_{r 4-3} \rho_{r 1-3} \rho_{t 3-4}^{2} v\left(t-2\left(\tau_{2}+\tau_{3}\right)\right)+ \\
& +\rho_{t 4}\left(\rho_{r 4-3} \rho_{r 1-3} \rho_{t 4-3}\right)^{2} \rho_{t 3-4}^{2} v\left(t-4\left(\tau_{2}+\tau_{3}\right)\right)+\cdots
\end{aligned}
$$

Os modelos deduzidos nesta seção para cada configuração foram posteriormente implementados no simulador. A seguir serão apresentadas as funcionalidades da interface do simulador proposto.

\section{Interface do simulador}

A Figura 12 apresenta a interface gráfica do simulador proposto. No elemento 1 destacado, é possível o usuário selecionar uma das quatro configurações propostas por meio de um botão de opções. No elemento gráfico 2 , o usuário entra com os dados elétricos dos trechos de linha correspondentes à configuração selecionada. No elemento gráfico 3, são oferecidos botões de opções para o usuário selecionar o trecho de linha e a barra (transmissora ou receptora) ao qual deseja analisar.

O cálculo das ondas viajantes no trecho configurado é feito após o usuário clicar no botão "CALCULAR" (elemento 4). No elemento 5 é disponibilizada uma área para plotagem da onda viajante no trecho selecionado. Para habilitar a plotagem do gráfico, o usuário deve clicar no botão "Plotar" (elemento 6). Adicionou-se ainda uma caixa de texto (elemento 7) para exibir a tensão máxima da onda viajante.

Na Seção 4 a seguir serão apresentados resultados demonstrativos obtidos utilizando o simulador proposto para duas das quatro configurações modeladas na Seção 3 .

\section{Resultados}

Para demonstração da aplicabilidade do simulador, foram realizadas simulações utilizando as configurações 1 e 3 . A seguir são apresentados e discutidos os resultados obtidos. 


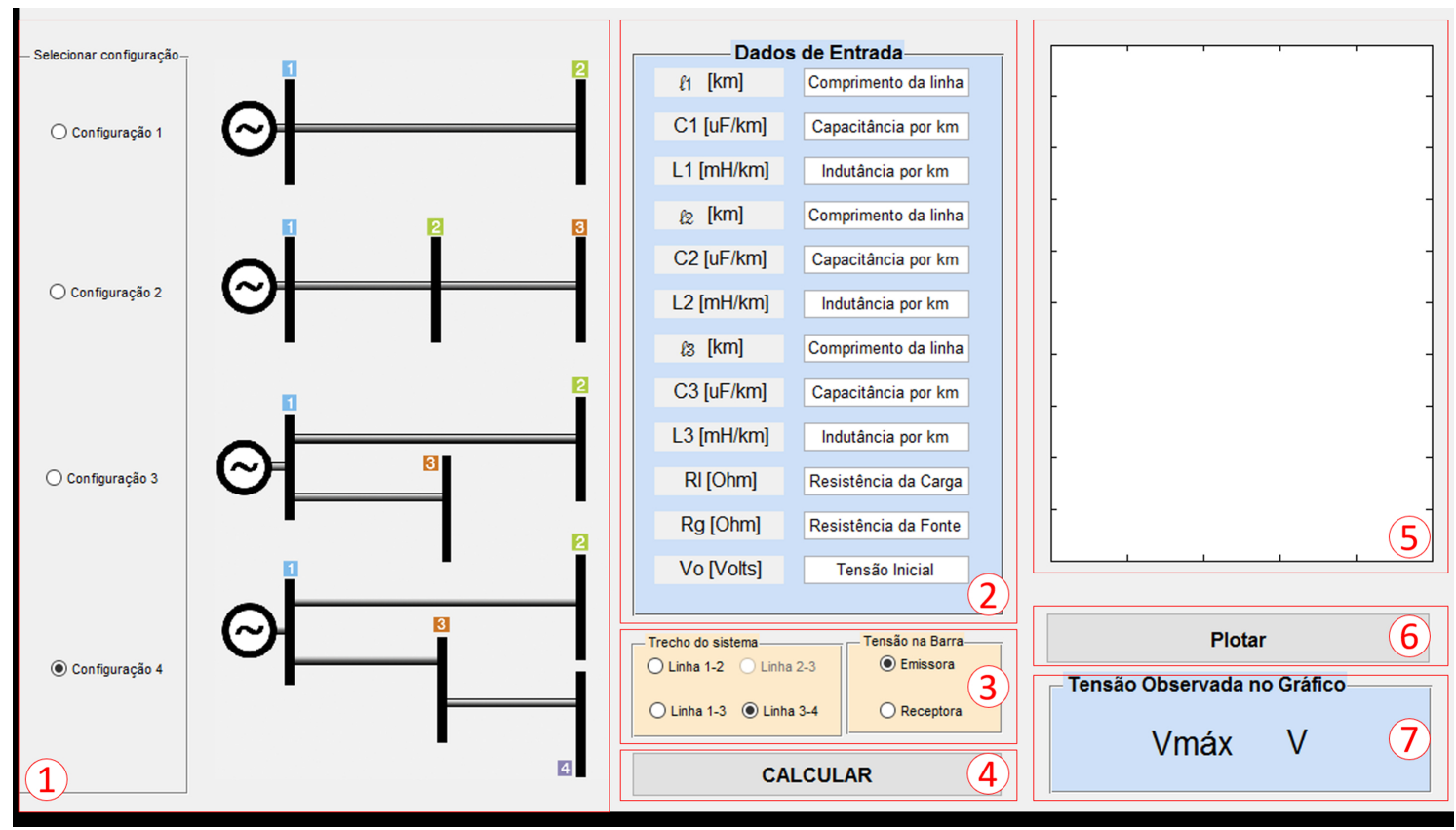

Figura 12: Tela do simulador didático de ondas viajantes.

\subsection{Configuração 1 (Linha 1-2)}

Para simulação da configuração 1, (com a linha 1-2 selecionada) foram utilizados os dados de linha apresentados na Tabela 1 e os parâmetros de propagação apresentados na Tabela 2.

Após a entrada dos dados e configuração do simulador, foram obtidos os resultados apresentados na Tabela 3 para as quatro primeiras reflexões.

Os resultados apresentados na Tabela 3 podem ser confirmados na Figura 13 para as sete primeiras reflexões. Nesta figura, a linha na cor vermelha representa a onda progressiva, enquanto a linha na cor azul representa a onda regressiva.

Tabela 1: Dados de entrada da configuração 1, linha 1-2.

\begin{tabular}{lc}
\hline Dado & Valor adotado \\
\hline $\mathbf{l}_{\mathbf{1}}$ & $15 \mathrm{~km}$ \\
$C_{1}$ & $6,25 \mathrm{~F} / \mathrm{km}$ \\
$L_{1}$ & $62,5 \mathrm{mH} / \mathrm{km}$ \\
$R_{L}$ & $900 \Omega$ \\
$R_{g}$ & $25 \Omega$ \\
$V_{0}$ & $1000 \mathrm{~V}$ \\
\hline
\end{tabular}

Tabela 2: Parâmetros de propagação da linha 1-2.

\begin{tabular}{lc}
\hline Parâmetro & Valor adotado \\
\hline$\tau_{1}$ & $50 \mu s$ \\
$\rho_{r 1-2}$ & 0,6 \\
$\rho_{t 1-2}$ & 1,6 \\
$\rho_{r 2-1}$ & $-0,6$ \\
$\rho_{t 2-1}$ & 0,4 \\
\hline
\end{tabular}

\subsection{Configuração 3 (Linha 1-2)}

Para a configuração 3, linhas 1-2 e 1-3, barra emissora, foram adotados os dados apresentados na Tabela 4. Para a linha 1-2, foram utilizados os parâmetros de propagação apresentados na Tabela 2, e para a linha 1-3, os parâmetros apresentados na Tabela 5 .

De posse dos dados de entrada e posterior configuração do simulador, procedeu-se com a simulação do sistema. Os resultados obtidos estão apresentados na Tabela 6 .

Para efeito comparativo, foram adicionados na Tabela 6 os resultados obtidos para as linhas 1-2 e 1-3 simuladas isoladamente, ou seja, na Configuração 1. Conforme

Tabela 3: Nível de tensão para a configuração 1, linha 1-2, barra emissora, para as sete primeiras reflexões.

\begin{tabular}{lcc}
\hline \multirow{2}{*}{ Reflexão } & \multicolumn{2}{c}{ Nível de tensão $(\mathrm{V})$} \\
\cline { 2 - 3 } & Onda Progressiva & Onda Regressiva \\
\hline $1^{\mathrm{a}}$ & $800 \mathrm{~V}$ & $640 \mathrm{~V}$ \\
$2^{\mathrm{a}} \mathrm{e} 3^{\mathrm{a}}$ & $-384 \mathrm{~V}$ & $-307,2 \mathrm{~V}$ \\
$4^{\mathrm{a}} \mathrm{e} 5^{\underline{\mathrm{a}}}$ & $184,32 \mathrm{~V}$ & $147,46 \mathrm{~V}$ \\
$6^{\mathrm{a}} \mathrm{e} 7^{\mathrm{a}}$ & $-88,47 \mathrm{~V}$ & $-70,78 \mathrm{~V}$ \\
\hline
\end{tabular}

Tabela 4: Dados de entrada para a configuração 3, linhas 1-2 e $1-3$.

\begin{tabular}{lc}
\hline Dado & Valor adotado \\
\hline $\mathbf{l}_{\mathbf{1}}$ & $15 \mathrm{~km}$ \\
$C_{1}$ & $10 \mathrm{~F}$ \\
$L_{1}$ & $6,25 \mathrm{mH}$ \\
$l_{2}$ & $30 \mathrm{~km}$ \\
$C_{2}$ & $5 \mathrm{~F}$ \\
$L_{2}$ & $3,125 \mathrm{mH}$ \\
$R_{L}$ & $100 \Omega$ \\
$R_{g}$ & $25 \Omega$ \\
$V_{0}$ & $1000 \mathrm{~V}$ \\
\hline
\end{tabular}




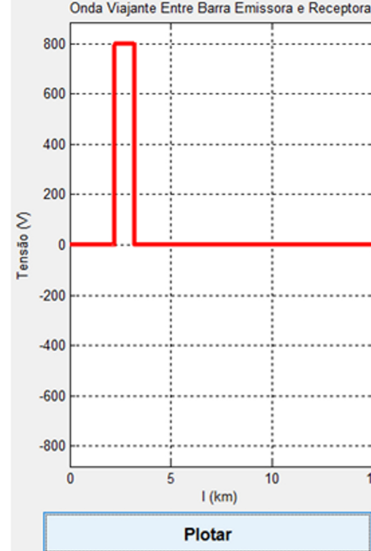

Tensão Observada no Gráfico 800

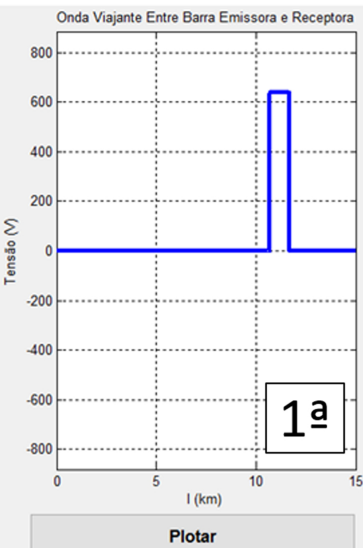

Tensão Observada no Gráfico

640

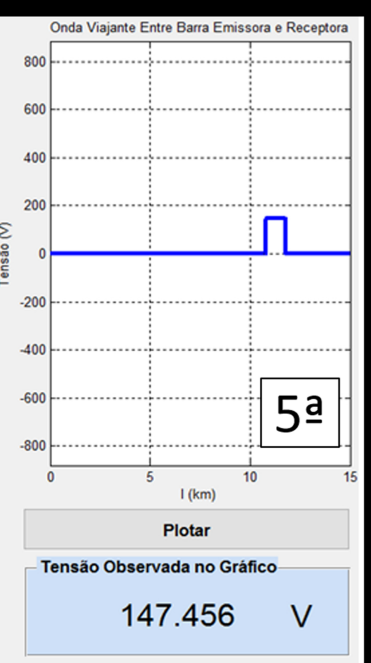

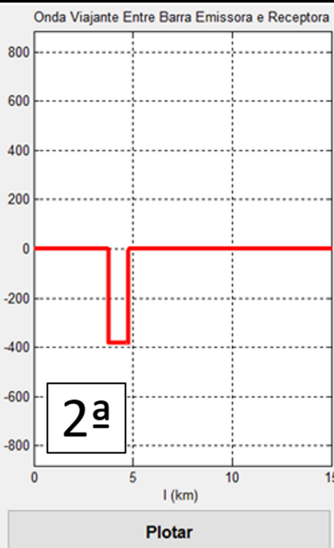

Tensão Observada no Gráfico $-384$
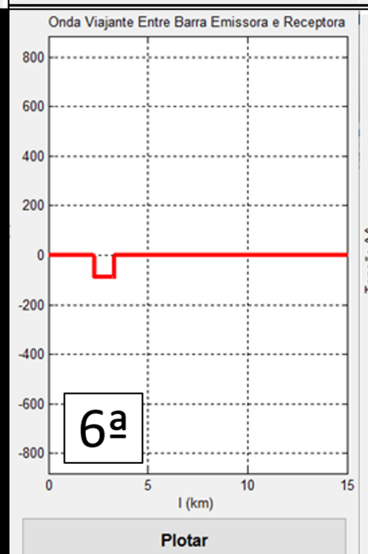

Tensão Observada no Gráfico

$-88.4736 \mathrm{~V}$

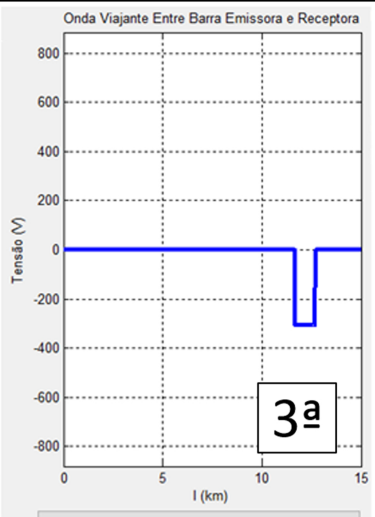

Plotar

Tensāo Observada no Gráfico $-307.2 \quad V$

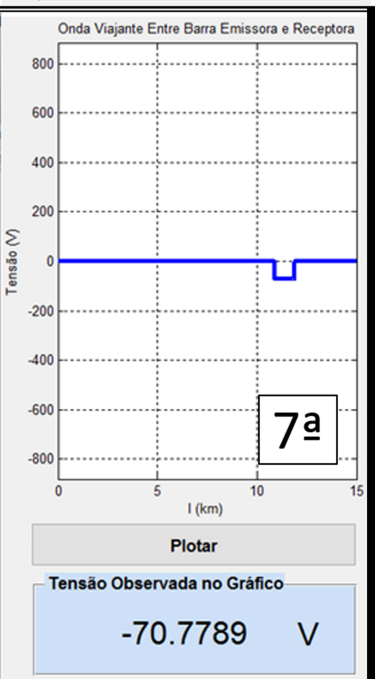

Figura 13: Resultado da simulação para a configuração 1, Linha 1-2.

definido anteriormente nas Tabelas 2 e 5 , tem-se que $\tau_{2}=2 \tau_{1}$, e que para a onda viajar o trecho 1-3 e voltar são necessários $200 \mu s$, ao passo que no trecho 1-2 é necessário metade desse tempo. Desse fato, decorre que a cada $200 \mu \mathrm{s}$ a onda viajante da linha 1-2 (L-12) sofre interferência da onda viajante da linha 1-3 (L-12). Na Tabela 6, a qual cada linha representa $50 \mathrm{~s}$, observa-se a interferência na quinta linha, a partir da qual os valores da terceira coluna diferenciam-se da primeira coluna.

A Figura 14 apresenta os resultados da simulação da Configuração 3 para a linha 1-2 diretamente no simulador. A linha na cor vermelha representa a onda progressiva, e a linha na cor azul representa a onda regressiva.

Tabela 5: Parâmetros de propagação da linha 1-3.

\begin{tabular}{lc}
\hline Parâmetro & Valor adotado \\
\hline$\tau_{2}$ & $100 \mu s$ \\
$\rho_{r 1-3}$ & -1 \\
$\rho_{t 1-3}$ & \\
$\rho_{r 3-1}$ & 1 \\
$\rho_{t 3-1}$ & 2 \\
\hline
\end{tabular}

Tabela 6: Ondas viajantes na Configuração 3 e análise comparativa com a Configuração 1 .

\begin{tabular}{lccc}
\hline Reflexão & $\begin{array}{c}\text { Tensão } \\
\text { em L1-2 } \\
\text { isolada (V) }\end{array}$ & $\begin{array}{c}\text { Tensão } \\
\text { em L1-3 } \\
\text { isolada (V) }\end{array}$ & $\begin{array}{c}\text { Tensão L1-2 } \\
\text { sob influência } \\
\text { de L1-3 (V) }\end{array}$ \\
\hline- & 1000 & 1000 & 1000 \\
$1^{\mathrm{a}}$ & -600 & 1000 & -600 \\
$2^{\mathrm{a}}$ & -360 & 1000 & -360 \\
$3^{\mathrm{a}}$ & 216 & 1000 & 216 \\
$4^{\mathrm{a}}$ & 129,60 & -1000 & 729,6 \\
$5^{\mathrm{a}}$ & $-77,76$ & -1000 & $-437,76$ \\
$6^{\mathrm{a}}$ & $-46,66$ & -1000 & $-262,66$ \\
$7^{\mathrm{a}}$ & 27,99 & -1000 & 157,59 \\
\hline
\end{tabular}

\section{Conclusões}

Neste trabalho foram estudadas diferentes configurações de sistemas de transmissão com enfoque na criação de um simulador de transitórios de ondas de tensão em linhas de transmissão, com a finalidade de tornar didático o processo de ensino-aprendizagem do estudo desse fenômeno por meio de animações feitas em uma interface gráfica. 


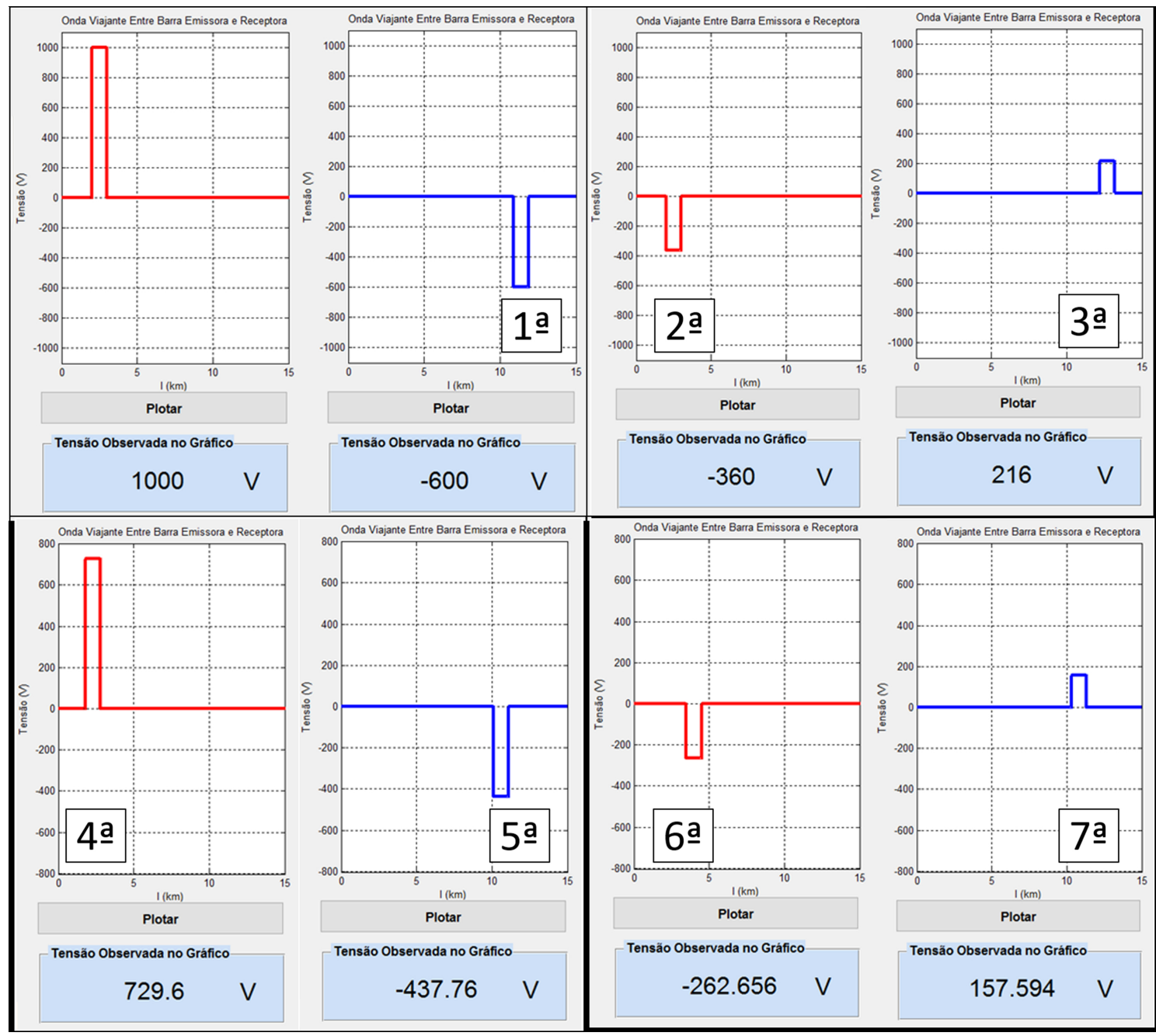

Figura 14: Resultados da simulação da configuração 3, linha 1-2.

O simulador desenvolvido constitui uma importante ferramenta para consolidação dos conhecimentos acerca dos transitórios de ondas tensão e como o fenômeno é similar à propagação de ondas eletromagnéticas em meios diferentes, também, proporciona um modo gráfico para melhor compreensão desse fenômeno. Ressalta-se ainda o aspecto inovador da ferramenta proposta, pois os softwares comerciais de análise de linhas de transmissão, apesar de fornecerem ferramentas para o estudo do comportamento do sistema nos períodos de transitório, no geral, não fornecem uma animação temporal, em especial para as ondas de tensão que viajam pelas linhas de transmissão.

Por fim, acrescenta-se que simulador desenvolvido, além de importante como apoio didático, pode funcionar como uma ferramenta de inclusão, pois, apesar do MATLAB não ser um software livre, o simulador criado com auxílio desse software baseia-se em um executável com todas as funcionalidades já mencionadas, e que pode ser instalado em qualquer computador que se utiliza do Windows como sistema operacional, sem a necessidade do MATLAB instalado.

\section{Referências}

[1] C.Y. Evrenosog̈lu, A. Abur e E. Akleman, Electric Power Systems Research 77, 876 (2006).

[2] D.D. Yong, C. Reineri e F. Magnago, IEEE Latin America Transactions 11, 479 (2013).

[3] T. Gonen. Modern power system analysis (CRC Press, Nova Iorque, 2016).

[4] H. Saadat, Power system analysis (McGraw-Hill, Nova Iorque, 1999).

[5] J.D. Glover, M. Sarma e T. Overbye, Power System Analysis $\&$ Design, SI Version (Cengage Learning, Stamford, 2012).

[6] W.D. Stevenson, Elements of power system analysis (McGraw-Hill, Nova Iorque, 1975).

[7] P.C. Magnusson, A. Weisshaar, V.K. Tripathi e G.C. Alexander, Transmission lines and wave propagation (CRC Press, Nova Iorque, 2000). 